\title{
On Circular Disarranged Strings of Sequences
}

\author{
F. Beggas ${ }^{1}$, M. M. Ferrari ${ }^{2}$, H. Kheddouci ${ }^{1}$, N. Zagaglia Salvi ${ }^{2}$ \\ 1 University of Lyon, LIRIS UMR5205 CNRS, Claude Bernard Lyon 1 University \\ 43 Bd du 11 Novembre 1918, F-69622, Villeurbanne, France \\ \{fairouz.beggas,hamamche.kheddouci\}@liris.cnrs.fr \\ 2 Dipartimento di Matematica, Politecnico di Milano \\ P.zza Leonardo da Vinci 32, 20133 Milano, Italy \\ \{margheritamaria.ferrari,norma.zagaglia\}@polimi.it
}

\begin{abstract}
Two sequences $\left(a_{1}, a_{2}, \ldots, a_{n}\right)$ and $\left(b_{1}, b_{2}, \ldots, b_{n}\right)$, sharing $n-1$ elements, are said disarranged if for every non-empty subset $Q \subseteq[n]$, the sets $\left\{a_{i} \mid i \in Q\right\}$ and $\left\{b_{i} \mid i \in Q\right\}$ are different. In this paper we investigate properties of these pairs of sequences. Moreover we extend the definition of disarranged pairs to a circular string of $n$-sequences and prove that, for every positive integer $m$, except some initials values for $n$ even, there exists a similar structure of length $m$.
\end{abstract}

Keywords: direct product of graphs, adjacent vertex distinguishing chromatic index, cyclic permutation, derangement, disarranged sequences, 1-disarranged sequences, circular disarranged string.

\section{Introduction}

Let $n$ be a positive integer, $[n]=\{1,2, \ldots, n\}$ and $R=\left(a_{1}, a_{2}, \ldots, a_{n}\right)$ and $S=\left(b_{1}, b_{2}, \ldots, b_{n}\right) n$-sequences of distinct elements, sharing exactly $n-1$ elements. We associate with $R$ and $S$ the bijection $f$ defined by the relation $f\left(a_{i}\right)=b_{i}, 1 \leq i \leq n$, and represented in two line notation by the $2 \times n$ array

$$
\left(\begin{array}{llll}
a_{1} & a_{2} & \ldots & a_{n} \\
b_{1} & b_{2} & \ldots & b_{n}
\end{array}\right)
$$

Let $u$ and $v$ be the different elements which belong to the first and the second line respectively. The function $f$ is formed by the linear ordering $l(f)=$ $\left(u, f(u), f^{2}(u), \ldots, f^{k-1}(u), v\right)$, where $k$ is the minimum positive integer such that $f^{k}(u)=v$, and a permutation $\pi(f)$ on the remaining elements. In [2] a similar function, called widened permutation, is investigated in the context of the theory of species of Joyal. A subset $H \subseteq R$ is $f$-fixed if $f(H)=H$. If we want emphasize the involved sequences, we denote the function $f$ by $f(R, S)$ and $l(f)$ by $l(R, S)$.

Definition 1. $R$ is said disarranged with respect to $S$ if for every set $\left\{i_{1}, i_{2}, \ldots, i_{r}\right\} \subseteq[n]\left\{a_{i_{1}}, a_{i_{2}}, \ldots, a_{i_{r}}\right\} \neq\left\{b_{i_{1}}, b_{i_{2}}, \ldots, b_{i_{r}}\right\}$. 
From the definition it follows that the relation is symmetric; then we say that $R$ and $S$ are disarranged. We also say that the pair $(R, S)$ and the function $f(R, S)$ are disarranged. The sequences $R$ and $S$ are called 1-disarranged if there exists an index $i \in[n]$ such that $a_{i}=b_{i}$ and the sequences, obtained from $R$ and $S$ after deleting $a_{i}$ and $b_{i}$, are disarranged. In this case we say that the pair $(R, S)$ is 1-disarranged.

Now we extend the definition 1 to a string of $n$-sequences.

Definition 2. Let $n, m \in \mathbb{N}$; an m-string $\left(S_{1}, S_{2}, \ldots, S_{m}\right)$ of $n$-sequences, is called disarranged if:

(A1) $S_{i}$ is disjoint from $S_{i-1}$ and $S_{i+1}$,

(A2) $S_{i-1}$ and $S_{i+1}$ are disarranged.

for every $i=2, \ldots, m-1$.

A disarranged $m$-string of $n$-sequences is circular when the properties (A1) and (A2) are satisfied for every $i=1,2, \ldots, m$ (taking the indices modulo $m)$.

An $m$-string of $n$-sequences is 1-disarranged if there exists at least one index $i$ such that $S_{i-1}$ or $S_{i+1}$ form a 1-disarranged pair.

The concatenation of two strings of sequences $P=\left(P_{1}, P_{2}, \ldots, P_{r}\right)$ and $T=$ $\left(T_{1}, T_{2}, \ldots, T_{q}\right)$, is the string $P T=\left(P_{1}, P_{2}, \ldots, P_{r}, T_{1}, T_{2}, \ldots, T_{q}\right)$. Assume that $P$ and $T$ are disarranged. If $P_{r}$ and $T_{1}$ are disjoint and in addition $\left(P_{r-1}, T_{1}\right)$ and $\left(P_{r}, T_{2}\right)$ are disarranged, then we have that $P T$ is disarranged. Moreover, if $P_{1}$ and $T_{q}$ are disjoint and the pairs $\left(P_{1}, T_{q-1}\right)$ and $\left(P_{2}, T_{q}\right)$ are disarranged, then $P T$ is a disarranged circular string. A similar definition holds in the case of 1-disarranged sequences.

The notion of circular disarranged or 1-disarranged string of $n$-sequences has application in relation to an edge coloring problem of graphs [5], where sequences of sets of colors which satisfy $(A 1)$ and $(A 2)$ are used; in [4] and [7] a similar structure is investigated.

Recall that an edge coloring of a finite simple undirected graph $G$ is a map $\alpha$ from the edge set $E(G)$ of $G$ to a finite set of colors $C$. The coloring $\alpha$ is proper if $\alpha\left(e_{1}\right) \neq \alpha\left(e_{2}\right)$ whenever edges $e_{1}, e_{2}$ are adjacent.

The color set of a vertex $u \in V(G)$ with respect to $\alpha$ is the set $C_{\alpha}(u):=\{\alpha(u v)$ : $u v \in E(G)\}$ of colors assigned by $\alpha$ to edges incident to $u$. The coloring $\alpha$ is adjacent vertex distinguishing (avd for short) if $u v \in E(G) \operatorname{implies} C_{\alpha}(u) \neq$ $C_{\alpha}(v)$. The adjacent vertex distinguishing chromatic index of the graph $G$ is the minimum number $\chi_{a}^{\prime}(G)$ of colors in a proper avd edge coloring of $G$.

The avd chromatic index was discussed also for graphs resulting from binary graph operations; good information about such operations are contained in [6]. In particular one can mention the direct product ([4], [7], [1]). The direct product of graphs $G$ and $H$ is the graph $G \times H$ with $V(G \times H):=V(G) \times V(H)$ and $E(G \times H):=\{(u, x)(v, y): u v \in E(G), x y \in E(H)\}$ (where $(u, x)(v, y)$ is 
a simplified notation for the undirected edge $\{(u, x),(v, y)\})$. This product is commutative and associative (up to isomorphisms).

In order to evaluate the avd chromatic index of the direct product of a simple graph $G$ by a cycle $C_{m}$, it is useful to employ the concept of a structure such as a circular disarranged or 1-disarranged string. The main result of this paper is the proof of the existence of circular disarranged strings of every length $m$, except three initial cases, where the strings are 1-disarranged.

We are interested in investigating disarranged pairs of sequences and the existence and construction of circular disarranged strings of sequences having equal length. This paper is organized as follows. In section 2 we determine a characterization of a pair of disarranged sequences. In section 3 we associate with a pair of disarranged sequences a permutation, which turns out to be formed by one cycle only; in the same section we determine many cases of circular disarranged strings. In sections 4 and 5 we prove by construction that for every positive integer $m$, there exist circular disarranged $m$-strings of $n$-sequences, $n>2$, of every length, except the cases of $n$ even and $m=6, m=14$ and $m=2 n+7$, where the strings are 1-disarranged. All these conclusive results are contained in the last section.

\section{Disarranged Pairs of Sequences}

In this section we determine a characterization of disarranged pairs of sequences of equal length.

Theorem 1. Let $R$ and $S$ be n-sequences, sharing exactly $n-1$ elements. They are disarranged if and only if the linear ordering $l(R, S)$ contains all the elements of $R$ and $S$.

Proof. Let $R=\left(a_{1}, a_{2}, \ldots, a_{n}\right)$ and $S=\left(b_{1}, b_{2}, \ldots, b_{n}\right)$ be $n$-sequences, sharing exactly $n-1$ elements. Assume they are disarranged.

If the function $f(R, S)$ contains a cycle $C=\left\{i_{1}, i_{2}, \ldots, i_{s}\right\}$, where $s>0$, then $f(C)=\left(f\left(i_{1}\right), f\left(i_{2}\right), \ldots, f\left(i_{s}\right)\right)=\left(i_{2}, i_{3}, \ldots, i_{1}\right)=C$. Then $C$ turns out to be $f$-fixed, a contradiction to the assumption. Thus $s=0$ and $f$ consists only in the linear ordering $l(f)$.

Now assume that the sequence $l(R, S)$ contains all the elements of $R$ and $S$. Then, for every subset $J$ of the set of elements of $R$, represented by the ordered subsequence of $l(f) J=\left(x_{1}, x_{2}, \ldots, x_{j}\right)$, where $j \geq 1$, we have that $f\left(x_{j}\right) \notin J$. This implies that $f(J) \neq J$; then $J$ is not $f$-fixed and consequently $R$ and $S$ are disarranged.

In other words $R$ and $S$ are disarranged if the function $f(R, S)$ is a widened permutation which consists only of the linear ordering $l(f)$.

A similar characterization holds in the case of two 1-disarranged sequences.

Proposition 1. Let $R=\left(a_{1}, a_{2}, \ldots, a_{n}\right)$ and $S=\left(b_{1}, b_{2}, \ldots, b_{n}\right)$ sequences, sharing exactly $n-1$ elements. They are 1-disarranged if and only if there exists 
an $i \in[n]$ such that $a_{i}=b_{i}$ and the linear ordering $l\left(R^{\prime}, S^{\prime}\right)$, where $R^{\prime}$ and $S^{\prime}$ are obtained from $R$ and $S$ by deleting $a_{i}$, contains all the elements of $R^{\prime}$ and $S^{\prime}$.

Example. Let $R=(1,2,3,4,5,6)$ and $S=(2,3,4,5,7,1)$, having different elements respectively 6 and 7 . The bijection $f(R, S)$ represented by the array

$$
\left(\begin{array}{llllll}
1 & 2 & 3 & 4 & 5 & 6 \\
2 & 3 & 4 & 5 & 7 & 1
\end{array}\right)
$$

is formed by the linear ordering $(6,1,2,3,4,5,7)$, which contains all the elements of $R \cup S$. Thus $R$ and $S$ are disarranged.

An example of 1-disarranged sequence is given by the two sequences $R=$ $(1,2,3,4,5,6,7)$ and $S=(7,4,3,1,6,8,5)$. In this case 3 is fixed, $u=2, v=8$

and the function $f(R, S)$ is partitioned into the cycle (3) and the linear ordering $(2,4,1,7,5,6,8)$.

\section{Disarranged Permutations}

In this section we introduce and investigate the notion of disarranged permutations to show the connection with the notion of disarranged pairs of $n$ sequences.

Definition 3. A permutation $\alpha$ of $[n]$ is said disarranged if every proper subset $D$ of $[n]$ is not $\alpha$-fixed.

Thus a disarranged permutation turns out to be a generalization of a derangement, which consists in a permutation without fixed points.

Recall that a permutation is cyclic if it consist of one cycle only [3].

Theorem 2. A permutation $\alpha$ of $[n]$ is disarranged if and only if $\alpha$ is cyclic.

Proof. Let $\alpha$ be a disarranged permutation of $[n]$. Assume that $\alpha$ has more than one cycle; in particular let $C=\left(j_{1}, j_{2}, \ldots, j_{s}\right)$ one of its cycles, where $s<n$. Then $\alpha(C)=\left(\alpha\left(j_{1}\right), \alpha\left(j_{2}\right), \ldots, \alpha\left(j_{s}\right)\right)=\left(j_{2}, j_{3}, \ldots, j_{s}, j_{1}\right)=C$, a contradiction to the assumption.

Now, assume that $\alpha$ has only one cycle, say $H$. Let $S=\left\{i_{1}, i_{2}, \ldots, i_{r}\right\}$, where $r<n$, a proper subset of $[n]$. Then $\alpha(S)=\left\{\alpha\left(i_{1}\right), \alpha\left(i_{2}\right), \ldots, \alpha\left(i_{r}\right)\right\}$ is the set of the elements of $H$ which are consecutive to the elements of $S$ in $H$. Because $r<n$, it follows that $\alpha(S) \neq S$ and $\alpha$ is disarranged.

Proposition 2. Let $R=\left(a_{1}, a_{2}, \ldots, a_{n}\right)$ and $S=\left(b_{1}, b_{2}, \ldots, b_{n}\right)$ sequences sharing the elements of $a(n-1)$-set $A$ and having $u$ and $v$ as different elements respectively. The function $f(R, S)$ is disarranged if and only if the function obtained by replacing $v$ by $u$ in $S$ is a cyclic permutation of $A \cup\{u\}$. 
Proof. Let $f$ be disarranged and let $l(f)=\left(u, a_{k_{1}}, a_{k_{2}}, \ldots, a_{k_{n-1}}, v\right)$. If we replace $v$ by $u$, we obtain a permutation of $A \cup\{u\}$, which is one cycle.

On the contrary, let $\pi$ be a permutation of $A \cup\{u\}$, formed by only one cycle, represented in two-line notation

$$
\left(\begin{array}{cccc}
a_{i_{1}} & a_{i_{2}} & \ldots & a_{i_{n}} \\
a_{i_{2}} & a_{i_{3}} & \ldots & a_{i_{1}}
\end{array}\right) .
$$

We replace the element $u$ by $v$ in the second line and we obtain a disarranged function.

Lemma 1. Let $\alpha$ be a permutation of $A=\left\{a_{1}, a_{2}, \ldots, a_{n}\right\}$ such that $\alpha\left(a_{i}\right)=$ $a_{i+k}\left(a_{i-k}\right)$, where $i \in[n], k$ is a positive integer coprime with $n$ and the integers $i+k(i-k)$ are modulo $n$. Then $\alpha$ is cyclic.

Proof. The permutation $\alpha$ contains the cycle $C=\left(a_{1}, a_{1+k}, \ldots, a_{1+(r-1) k}\right)\left(C^{\prime}=\right.$ $\left.\left(a_{1}, a_{1-k}, \ldots, a_{1-(r-1) k}\right)\right)$, where $r$ is the minimum positive integer such that $1+r k \equiv 1(1-r k \equiv 1) \bmod n$. Because $k$ is prime with $n$, then $r=n$ and $C$ $\left(C^{\prime}\right)$ contains all the elements of $A$.

Let $R$ and $S$ be $n$-sequences sharing $n-1$ elements; $u$ and $v$ are the different elements respectively. For every $k \in[n-1]$ we say that $S$ is a $k$-right (k-left) shift of $R$ if $S$ is obtained from $R$ by cyclically shifting of $k$ positions to the right (left) all the elements of $R$ and replacing $u$ by $v$.

Moreover $S$ is a $k$-shift of $R$ when it is either a $k$-right or a $k$-left shift of $R$. Given the permutation $\sigma=(1,2, \ldots, n)$ of $[n]$, a $k$-left shift of $R=\left(a_{1}, a_{2}, \ldots, a_{n}\right)$ is $\sigma^{k}(R)=\left(a_{1+k}, a_{2+k}, \ldots, a_{n+k}\right)$, while a $k$-right shift of $R$ is $\sigma^{-k}(R)=$ $\left(a_{1-k}, a_{2-k}, \ldots, a_{n-k}\right)$, where the indices are modulo $n$.

Proposition 3. Let $R$ and $S$ be $n$-sequences sharing $n-1$ elements and let $S$ be a $k$-shift of $R$, where $k$ is a positive integer coprime with $n$. Then $(R, S)$ is disarranged.

Proof. It follows from Lemma 1 and Proposition 2.

An example of application of Proposition 3 is given from the circular disarranged string of $n$-sequences of length $2 n+1, n>2$, of the following Proposition.

Proposition 4. Let $n>2$ be a positive integer. Then the circular string

$$
C_{2 n+1}=\left(Q_{1}, Q_{2}, \ldots, Q_{2 n+1}\right),
$$

where $Q_{1}=\{1,2, \ldots, n\}, Q_{2}=\{n+1, n+2, \ldots, 2 n\}$ and every other sequence $Q_{i}, 3 \leq i \leq 2 n+1$, is obtained from $Q_{i-1}$ by taking $n$ cyclically consecutive elements of the sequence $\{1,2, \ldots, 2 n+1\}$, is disarranged.

Proof. By construction, every $Q_{i}$ is 1 -right shift of $Q_{i-2}, 3 \leq i \leq 2 n+1$ and by Proposition 3 the result is done. 
Lemma 2. Let $R=P_{1} P_{2} \ldots P_{t}$ be a concatenation of $d$-sequences, $d, t>1$. Then the sequences $S=P_{2} P_{3} \ldots P_{t} \sigma^{ \pm k}\left(P_{1}\right)$ and $S^{\prime}=\sigma^{ \pm k}\left(P_{t}\right) P_{1} P_{2} \ldots P_{t-1}$, where $k$ is a positive integer coprime with $d$, form together with $R$ a cyclic permutation.

Proof. Let $P_{i}=\left(p_{i, 1}, p_{i, 2}, \ldots, p_{i, d}\right), 1 \leq i \leq t$, and denote $V_{j}=\left(p_{1, j}, p_{2, j}, \ldots, p_{t, j}\right)$, where $1 \leq j \leq d$.

Note that, since $k$ is coprime with $d$, the set $\left\{p_{1,1}, p_{1,1+k}, \ldots, p_{1,1+(d-1) k}\right\}$ coincides with $P_{1}$. Thus the permutation having $R$ and $S$ as rows in the line notation

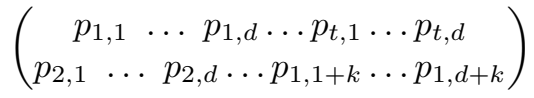

is formed by the cycle obtained by the concatenation $V_{1} V_{1+k} V_{1+2 k} \ldots V_{1+(d-1) k}$, which contain all the elements of $R$. A similar situation holds for the pair $R$ and $S^{\prime}$.

Proposition 5. Let $R=P_{1} P_{2} \ldots P_{t}$ be a concatenation of d-sequences, $d, t>1$, $S=P_{2} P_{3} \ldots P_{t} \sigma^{ \pm k}\left(P_{1}\right)$ and $S^{\prime}=\sigma^{ \pm k}\left(P_{t}\right) P_{1} P_{2} \ldots P_{t-1}$, where $k$ is a positive integer coprime with $d$. If an element $x$ of $R$ is replaced by $y$ in $S$ or in $S^{\prime}$, $y \neq x$, then the new pairs of sequences are disarranged.

Proof. It follows from Lemma 2 and Proposition 2.

Lemma 3. Let $n$ be a positive even integer, $R=(1,2, \ldots, n)$ and $S=(3,4, \ldots, n, x, 1)$, where $x \notin R$. Then $(R, S)$ is disarranged.

Proof. It is easy to prove that $l(R, S)=(2,4, \ldots, n, 1,3, \ldots, n-1, x)$ contains all the elements of $R$ and $S$.

Lemma 4. Let $n$ be a positive even integer, $R=(1,2, \ldots, n)$ and $S=(3,4, \ldots, n, 2, y)$, where $y \notin R$. Then $(R, S)$ is disarranged.

Proof. As in the previous lemma $l(R, S)=(1,3, \ldots, n-1,2,4, \ldots, n, y)$ contains all the elements of $R$ and $S$.

\section{Circular Disarranged Strings of Even Length}

In this section we determine a construction of circular disarranged $m$-strings of $n$-sequences, where $n$ is a positive integer and $m \in\{8,10,12\}$, whose elements belong to the set $[2 n+1]$.

Throughout this section and the following ones we apply properties of Proposition 3 and Proposition 5 without an explicit recall.

Moreover if we want to remark the different elements $u$ and $v$ of a disarranged ordered pair of sequences, we write $[u \hookrightarrow v]$.

Recall that $Q_{i}, 1 \leq i \leq 2 n+1$, denotes the $i$-th sequence of the circular string $C_{2 n+1}$ of Proposition 4 . 
Proposition 6. Let $n$ be a positive integer. Then the circular 8-string of $n$ sequences

$E_{8}^{n}: T_{i}=Q_{i}, 1 \leq i \leq 5, T_{6}=(n+1, \ldots, 2 n-1, n-1), T_{7}=(n, 1, \ldots, n-2,2 n)$,

$$
T_{8}=(2 n+1, n+1, \ldots, 2 n-1)
$$

is disarranged.

Proof. By Proposition 4 the sequences $T_{3}, T_{4}, T_{5}$ are 1 -right shift of $T_{1}, T_{2}, T_{3}$ respectively.

Moreover it is easy to see that $T_{6}$ is 1-left shift of $T_{4}=(n, n+1, \ldots, 2 n-1)$ $([n \hookrightarrow n-1]), T_{7}$ is 1 -left shift of $T_{5}=(2 n, 2 n+1,1,2, \ldots, n-2)([2 n+1 \hookrightarrow n])$ and 1-right shift of $T_{1}([n-1 \hookrightarrow 2 n])$ and, finally, $T_{8}$ is 1-right shift of $T_{6}([n \hookrightarrow$ $2 n+1])$ and $T_{2}([2 n \hookrightarrow 2 n+1])$.

Proposition 7. Let $n$ be a positive odd integer. Then the circular 10-string of $n$-sequences

$$
\begin{aligned}
& \qquad E_{10}^{n}: Z_{i}=Q_{i}, 1 \leq i \leq 4, Z_{5}=(1,2, \ldots, n-1,2 n), \\
& Z_{6}=(2 n+1, n+2, \ldots, 2 n-1, n), Z_{7}=(2, \ldots, n-1,2 n, n+1), \\
& Z_{8}=(n+2, \ldots, 2 n-1,1,2 n+1), Z_{9}=(3, \ldots, n+1,2), Z_{10}=(2 n, 2 n+1, n+2, \ldots, 2 n-1), \\
& \text { is disarranged, }
\end{aligned}
$$

Proof. By Proposition 4 the sequences $Z_{3}, Z_{4}$ are 1-right shift of $Z_{1}, Z_{2}$ respectively. Moreover the sequences $Z_{i}$, for $5 \leq i \leq 8$ are 1-left shift of $Z_{i-2}, Z_{9}$ is 1-left shift of $Z_{7}$ and 2-left shift of $Z_{1}$. Finally $Z_{10}$ is 2-right shift of $Z_{8}$ and 1-right shift of $Z_{2}$. Note that because $n$ is odd, then 2 is coprime with $n$.

Proposition 8. Let $n=2 h$ be a positive even integer, $h>2$. Then the circular 10-string of $n$-sequences

$$
\begin{gathered}
E_{10}^{\prime n}: Z_{i}^{\prime}=Q_{i}, 1 \leq i \leq 4, Z_{5}^{\prime}=(1,2, \ldots, n-1,2 n), \\
Z_{6}^{\prime}=(2 n+1, n+2, \ldots, 2 n-1, n), Z_{7}^{\prime}=(h+1, \ldots, n-1, n+1 ; h, 1,2, \ldots, h-1), \\
Z_{8}^{\prime}=(n+h+1, \ldots, 2 n-1, n, n+2, \ldots, n+h, 2 n), \\
Z_{9}^{\prime}=(n, 1,2, \ldots, h-1, h+2, n-1, n+1, h+1), \\
Z_{10}^{\prime}=(n+2, \ldots, n+h, 2 n, 2 n+1, n+h+1, \ldots, 2 n-1),
\end{gathered}
$$

is disarranged. 
Proof. Recall that the sequences $Z_{3}^{\prime}, Z_{4}^{\prime}$ are 1-right shift of $Z_{1}^{\prime}, Z_{2}^{\prime}$ respectively. The sequence $Z_{5}^{\prime}$ is 1-left shift of $Z_{3}^{\prime}$. Moreover, denoted $Z_{5}^{\prime}=P_{1} P_{2}$, where $P_{1}=(1,2, \ldots, h), P_{2}=(h+1, \ldots, 2 h-1,2 n)$, we obtain $Z_{7}^{\prime}=P_{2} \sigma^{-1}\left(P_{1}\right)$, with $[2 n \hookrightarrow n+1]$. Now, denoted $Z_{7}^{\prime}=R_{1} R_{2}$, where $R_{1}=(h+1, \ldots, n-1, n+1)$ and $R_{2}=(h, 1,2, \ldots, h-1)$ we have $Z_{9}^{\prime}=R_{2} \sigma\left(R_{1}\right)=(n, 1, \ldots, h-1 ; h+2, \ldots, n-$ $1, n+1, h+1)$ with $[h \hookrightarrow n]$. Then $\left(Z_{9}^{\prime}, Z_{1}^{\prime}\right)=(n+1, n-1, \ldots, h+1, n, 1,2, \ldots, h)$ contains all the elements of $Z_{1}^{\prime}$ and $Z_{9}^{\prime}$.

Now we establish that $Z_{6}^{\prime}=(2 n+1, n+2, \ldots, 2 n-1, n)$ is 1-left shift of $Z_{4}=$ $(n, \ldots, 2 n-1)$ with $[n+1 \hookrightarrow 2 n+1]$.

Denoted $Z_{6}^{\prime}=A_{1} A_{2}$, where $A_{1}=(2 n+1, n+2, \ldots, n+h)$ and $A_{2}=(n+h+$ $1, \ldots 2 n-1, n)$, then $Z_{8}^{\prime}=A_{2} \sigma\left(A_{1}\right)=(n+h+1, \ldots, 2 n-1, n ; n+2, \ldots, n+$ $h, 2 n)=T_{1} T_{2}$ with $[2 n+1 \hookrightarrow 2 n]$. It follows $Z_{10}^{\prime}=T_{2} \sigma^{-1}\left(T_{1}\right)=(n+2, \ldots, n+$ $h, 2 n ; 2 n+1, n+h+1, \ldots, 2 n-1)$ with $[n \hookrightarrow 2 n+1]$. Finally $\left(Z_{10}^{\prime}, Z_{2}^{\prime}\right)=$ $(2 n+1, n+h+1, \ldots, 2 n, n+h, \ldots, n+1)$, where the sequence contains all the elements of $Z_{10}^{\prime}$ and $Z_{2}^{\prime}$.

By all previous motivations the string $E_{10}^{\prime n}$ is disarranged.

For $n \geq 3$, consider the $(n-1)$-sequences $A=\{1,2, \ldots, n-1\}$ and $B=$ $\{n+1, n+2, \ldots, 2 n-1\}$ of the set $[2 n+1]$; for every $x \in\{n, 2 n, 2 n+1\}$ denote $A x$ the ordered set obtained by inserting $x$ after last element of $A$. A similar definition holds for $x A, B x$ and $x B$.

The following sequence is an example of disarranged 6 -string of $n$-sequences:

$$
P_{1}=(A n, B 2 n,(2 n+1) A, n B, A 2 n, B(2 n+1)) .
$$

Indeed the third and the fourth sequences are 1-right shift of the first and the second respectively, while the fifth and the sixth are 1-left shift of the third and the fourth respectively.

A similar situation holds for the following 6 -string of $n$-sequences.

$$
P_{2}=(n A, 2 n B, A(2 n+1), B n, 2 n A,(2 n+1) B) .
$$

From previous disarranged strings it is immediate to obtain the following circular disarranged string.

Proposition 9. The concatenation $P_{1} P_{2}$ is a disarranged circular 12-string of $n$-sequences.

Example 1. For $n=3$, we have the following disarranged 6 -strings of 3 -sequences

$$
\begin{aligned}
& P_{1}=(123,456,712,345,126,457) \\
& P_{2}=(312,645,127,453,612,745)
\end{aligned}
$$

and the following circular disarranged 12-string of 3-sequences

$$
P_{1} P_{2}=(123,456,712,345,126,457,312,645,127,453,612,745) .
$$




\subsection{The circular strings of length 6 and 14}

Now we consider the particular case of circular strings of length 6 or 14 of $n$-sequences.

Proposition 10. Let $n$ be a positive integer. Then, for $n$ odd, the circular 6 string

$E_{6}^{n}: V_{i}=Q_{i}, 1 \leq i \leq 4, V_{5}=(2, \ldots, n-1,2 n, 1), V_{6}=(n+2, \ldots, 2 n-1,2 n+1, n+1)$,

is disarranged, while, for $n$ even, the circular 6 -string

$E_{6}^{\prime n}: V_{i}, 1 \leq i \leq 4, V_{5}^{\prime}=(1, n-1,2 n, 2, \ldots, n-2), V_{6}^{\prime}=(n+1,2 n-1,2 n+1, n+2, \ldots, 2 n-2)$,

is 1-disarranged. In particular the sequences $V_{1}$ and $V_{5}^{\prime}$ have the element 1 fixed, while $V_{2}$ and $V_{6}^{\prime}$ have $n+1$ fixed.

Proof. Let $n$ be odd. By Proposition $4\left(V_{3}, V_{1}\right)$ and $\left(V_{4}, V_{2}\right)$ are disarranged; moreover $V_{5}$ and $V_{6}$ are 1-left shift of $V_{1}$ and $V_{2}$ respectively, while they are 2-left shift of $V_{3}$ and $V_{4}$ respectively. Being 2 coprime with $n$, by Proposition 3 $\left(V_{3}, V_{5}\right)$ and $\left(V_{4}, V_{6}\right)$ are disarranged. By Lemmas 3 and $4 E_{6}^{n}$ is disarranged. Let $n$ be even. By the same previous motivation $\left(V_{3}, V_{1}\right)$ and $\left(V_{4}, V_{2}\right)$ are disarranged. Moreover $V_{5}^{\prime} \backslash\{1\}$ and $V_{6}^{\prime} \backslash\{n+1\}$ are 2-right shift of $V_{1} \backslash\{1\}$ and $V_{2} \backslash\{n+1\}$ respectively. Finally we have that the sequence

$$
l\left(V_{3}, V_{5}^{\prime}\right)=(2 n+1,1, n-1, \ldots, 2,2 n)
$$

contains all the elements of $V_{3}$ and $V_{5}^{\prime}$ and the sequence

$$
l\left(V_{4}, V_{6}^{\prime}\right)=(n, n+1,2 n-1, \ldots, n+2,2 n+1)
$$

contains all the elements of $V_{4}$ and $V_{6}^{\prime}$. By Theorem 1 the pairs $\left(V_{3}, V_{5}^{\prime}\right)$ and $\left(V_{4}, V_{6}^{\prime}\right)$ are disarranged.

Now let us consider the case of $m=14$. Since $14=8+6$, by concatenating a circular disarranged string of length 8 from Proposition 6 and a 1-disarranged string of length 6 from Proposition 10, we obtain that there exists a 1-disarranged string of length 14 .

Particular case. Take the case $n=4$. From the circular strings $E_{8}^{4}$ of Proposition 6 and the circular 1-disarranged string $E_{6}^{\prime 4}$ of Proposition 10 we obtain the circular strings $E_{6}^{\prime 4}:(1234,5678,9123,4567,1382,5796)$ and $E_{8}^{\prime 4}:(1234,5678,9123,4567,8912,5673,4128,9567)$.

Note that $E_{6}^{\prime 4}$ is 1-disarranged. Indeed last two strings have the elements 1 and 5 fixed with respect the first and the second sequence respectively. By concatenation we obtain the 1-disarranged circular string $E_{14}^{\prime 4}$ : $(1234,5678,9123,4567$, $8912,5673,4128,9567,1234,5678,9123,4567,1382,5796)$. 


\section{Circular Disarranged Strings of Odd Length}

In this section we give a construction of circular disarranged $m$-strings of $n$-sequences, where $n>2$ and $m \in\{2 n+3,2 n+5\}$. Recall that by Proposition 4 there exists a circular disarranged $(2 n+1)$-string of $n$-sequences, denoted $Q_{i}$, $1 \leq i \leq 2 n+1$.

Proposition 11. For $n>4$ be a positive integer. Then, for $n$ odd, the circular string of length $(2 n+3)$

$$
C_{2 n+3}=\left(Q_{1}, \ldots, Q_{2 n-1}, W_{2 n}, W_{2 n+1}, W_{2 n+2}, W_{(2 n+3)}\right),
$$

where $W_{2 n}=(n+2,3, \ldots, n, 1), W_{2 n+1}=(2, n+3, \ldots, 2 n+1), W_{2 n+2}=$ $(3,4, \ldots, n, 1, n+1)$ and $W_{2 n+3}=(n+3, \ldots, 2 n+1, n+2)$, and, for $n>4$, even, the circular string

$$
C_{2 n+3}^{\prime}=\left(Q_{1}, \ldots, Q_{2 n-3}, W_{2 n-2}^{\prime}, W_{2 n-1}^{\prime}, W_{2 n}^{\prime}, W_{2 n+1}^{\prime}, W_{2 n+2}^{\prime}, W_{2 n+3}^{\prime}\right),
$$

where $W_{2 n-2}^{\prime}=(3,4, \ldots, n+2) W_{2 n-1}^{\prime}=(n+5, \ldots, 2 n+1,1, n+3, n+4)$ $W_{2 n}^{\prime}=(4,5, \ldots, n+1,2,3) W_{2 n+1}^{\prime}=(n+4, \ldots, 2 n+1,1, n+2) W_{2 n+2}^{\prime}=$ $(3, \ldots, n+1,1), W_{2 n+3}^{\prime}=(n+2, n+3, \ldots, 2 n+1, n+3)$, are disarranged.

Proof. Let $n$ be odd. We see that $W_{2 n}$ is 1-right shift of $Q_{2 n-2}=(3, \ldots, n+2)$, $W_{2 n+2}$ is 1-left shift of $W_{2 n}$ and it is 2-left shift of $Q_{1}$.

Moreover $W_{2 n+1}$ is 1-right shift of $Q_{2 n-1}=(n+3, \ldots, 2 n+1,1), W_{2 n+3}$ is 1-left shift of $W_{2 n+1}$ and it is 2-left shift of $Q_{2}$.

Let $n$ be even. Recall that $Q_{2 n-4}=(4,5, \ldots, n+2)$ and $Q_{2 n-3}=(n+4, \ldots, 2 n+$ $1,1,2)$; and We have that $W_{2 n-2}^{\prime}$ is 1-right shift of $W_{2 n-4}^{\prime}$ with $n+3 \hookrightarrow 3, W_{2 n}^{\prime}$ is 1-left shift of $W_{2 n-2}^{\prime}$ with $n+2 \hookrightarrow 2, W_{2 n+2}^{\prime}$ is 1-right shift of $W_{2 n}^{\prime} 2 \hookrightarrow 1$. Moreover $W_{2 n-1}^{\prime}$ is 1-left shift of $W_{2 n-3}^{\prime}$ with $2 \hookrightarrow n+3, W_{2 n+1}^{\prime}$ is 1-right shift of $W_{2 n-1}^{\prime}$ with $2 \hookrightarrow n+3, W_{2 n+3}^{\prime}$ is 1 -right shift of $W_{2 n+1}^{\prime}$ with $1 \hookrightarrow n+3$.

Moreover $W_{2 n+2}^{\prime}$ and $Q_{1}$ are disarranged because there is the linear ordering $(n+1, n-1, \ldots, 1, n, n-2, \ldots, 2)$ which contain all the elements of $W_{2 n+2}^{\prime}$ and $Q_{1}$.

Finally $W_{2 n+3}^{\prime}$ and $Q_{2}$ are disarranged because there is the linear ordering $(2 n+$ $1,2 n-1, \ldots, n+3,2 n, 2 n-2, \ldots, n+2, n+1)$ which contain all the elements of $W_{2 n+3}^{\prime}$ and $Q_{2}$.

Proposition 12. For $n>4$, the circular string

$$
C_{2 n+5}=\left(U_{1}, \ldots, U_{2 n}, U_{2 n+1}, U_{2 n+2}, U_{2 n+3}, U_{2 n+4}, U_{2 n+5}\right),
$$

where $U_{i}=Q_{i}, 1 \leq i \leq 2 n$,

$$
\begin{gathered}
U_{2 n+1}=(1, n+3, \ldots, 2 n, n+2), U_{2 n+2}=(2 n+1,2, \ldots, n) \\
U_{2 n+3}=(n+2,1, n+3, \ldots, 2 n-1, n+1), U_{2 n+4}=(2, \ldots, n, 2 n), \\
\left.U_{2 n+5}=(2 n+1, n+3, \ldots, 2 n-1, n+1, n+2)\right),
\end{gathered}
$$

is disarranged. 
Proof. Recall that $U_{2 n-1}=(n+3, \ldots, 2 n+1,1)$ and $U_{2 n}=(2, \ldots, n+1)$. It is easy to prove that $U_{i}$, for $3 \leq i \leq 2 n+3$ is a 1-right shift of $U_{i-2}, U_{2 n+5}$ is 1-left shift of $U_{2 n+3}$ and compared with $U_{2}$ determines the sequence

$$
l\left(U_{2}, U_{2 n+5}\right)=(2 n, n+2, n+3, \ldots, 2 n-1, n+1,2 n+1)
$$

which contains all the elements of $U_{2}$ and $U_{2 n+5}$. By Theorem $1\left(U_{2}, U_{2 n+5}\right)$ is disarranged. Moreover $U_{2 n+4}$ is 1-left shift of $U_{2 n+2}$ and of $U_{1}$.

\section{Conclusive results}

In this last section we summarize previous results; in particular we prove the existence of circular disarranged $m$-strings, for every positive integer $m>2$, except few initial values.

Lemma 5. Let $m>6$ and $m \neq 14$ be a positive even integer. Then $m$ may be represented as integral linear combination of the integers 8, 10,12.

Proof. It is sufficient to prove that every integer $10 a \leq m \leq 10(a+1)$, where $a$ is a positive integer, satisfy the request. It is immediate that the integers 16,18 , $10 a, 10 a+8,10(a+1)$ satisfy the condition. Assume that $a \geq 2$. We write the integers $10 a+2,10 a+4,10 a+6$, as $10(a-1)+12,10(a-2)+24$ and $10(a-1)+16$ respectively, thus proving the result.

Proposition 13. Let $m$ be a positive even integer and $n$ a positive integer. For every $m \geq 8$ and $m \neq 14$, if $n$ is even, and $m \geq 4$, if $n$ is odd, there exists a circular disarranged $m$-string of $n$ sequences. For $m=6$ and $m=14$ there exists a circular 1-disarranged m-string of $n$ sequences.

Proof. By Propositions 6, 7, 8 and 9, there are circular disarranged sequences for $m=8,10,12$. By Lemma 5 for every even integer $m \geq 8$ and $m \neq 14$, by concatenating suitable strings whose elements belong to $[2 n+1]$, we obtain a circular disarranged $m$-string. In the remaining initial cases we have a 1-disarranged $m$-string.

Lemma 6. Let $m \geq 2 n+1$ be a positive odd integer, where $n \geq 3$. Then $m$ may be represented as sum of $2 n+1$ or $2 n+5$ and an even integer $k \geq 8$.

Proof. Every integer $h=2 n+h^{\prime}$, where $h^{\prime} \geq 9$ is odd may be represented as $h=2 n+1+t$, where $t \geq 8$ is a positive even integer. If $t=14$, we write $h=2 n+5+10$.

Proposition 14. Let $m \geq 2 n+1$, where $n \geq 3$, be a positive odd integer. Then there exists a circular disarranged $m$-string of $n$ sequences, except $m=2 n+7$ where there exists a 1-disarranged circular string of $n$ sequences.

Proof. By Lemma 6 every integer $m$, except $m=2 n+7$, is a sum of $2 n+1$ or $2 n+5$ and an even integer $\geq 8$. Then by concatenating suitable strings we obtain the result. In the case of $m=2 n+7$ by concatenating a $(2 n+1)$-string and a circular 6 -string, we obtain a circular 1-disarranged $m$-string. 
In conclusion we obtain the following result.

Theorem 3. Let $m, n$ be positive integers. For $n$ odd and every $m>2$ or for $n$ even and $m>6$ even $(m \neq 14)$ or for $m \geq 2 n+1$ odd $(m \neq 2 n+7)$, there exists a circular disarranged $m$-string. For the remaining cases, there exists a circular 1-disarranged m-string.

A further development concerns the problem of establishing whether some 1-disarranged strings are disarranged.

\section{References}

1. J.-L. Baril, H. Kheddouci, O. Togni, Vertex distinguishing edge- and total-colorings of Cartesian and other product graphs, Ars Combinatoria, 107 (2012), 109-127.

2. F. Beggas, M. M. Ferrari, N. Zagaglia Salvi, Combinatorial interpretations and enumeration of particular bijections, Riv.Mat.Univ.Parma, (2016), to appear.

3. M. Bona, Combinatorics of Permutations, Chapman and Hall/CRC Press, Boca Raton, FL, (2004).

4. L. Frigerio, F. Lastaria, N. Zagaglia Salvi, Adjacent vertex distinguishing edge colorings of the direct product of a graph by a path or a cycle, Discussiones Mathematicae Graph Theory, 31 (2011), 547 - 557.

5. M. Horňák, D. Mazza and N. Zagaglia Salvi, Edge colorings of the direct product of two graphs, Graphs and Combinatorics, 31 (2015), 975 - 992 .

6. W. Imrich and S. Klavžar, Product Graphs: Structure and Recognition, WileyInterscience, New York (2000).

7. E. Munarini, C. Perelli Cippo, N. Zagaglia Salvi, On the adjacent vertex distinguishing edge colorings of direct product of graphs, Recent results in designs and graphs: a Tribute to Lucia Gionfriddo, vol. 28, Quaderni di Matematica, Aracne Ed., (2013), 369-392. 